\title{
Synthesis of novel urethanes from a castor oil derived C22-acyloin
}

\author{
Marijke Van der Steen, ${ }^{\text {a }}$ Maris Vilums, and Christian V. Stevens ${ }^{\mathrm{a} *}$ \\ ${ }^{a}$ Department of Sustainable Organic Chemistry and Technology, Faculty of Bioscience \\ Engineering, Ghent University, Coupure Links 653, 9000 Ghent, Belgium \\ E-mail: chris.stevens@ugent.be
}

\begin{abstract}
The synthesis of new bio-based chemical building blocks, resulting from the condensation of a renewable C-22 acyloin derived from non-edible castor oil, with mono- and bifunctional isocyanates is reported. The condensation with aliphatic mono-isocyanates was relatively straightforward, however phenyl isocyanates only resulted in low yields together with the formation of a cyclic hemi-aminal during purification. The condensation with diisocyanates was successful for the aliphatic hexamethylene diisocyanate. As for the aromatic 2,4-toluene diisocyanate, a low yield of the desired product was obtained, since a similar ring closing reaction took place. The urethanes were synthesized in order to evaluate their plasticizing and viscosity-modifying properties.
\end{abstract}

Keywords: Acyloin, castor oil, isocyanates, nucleophilic addition, undecylenic acid, urethanes

\section{Introduction}

Depletion of fossil resources and the effects of climate change provide an impulse for the development of sustainable fuels and chemicals from renewable resources. Novel platform chemicals are being developed or rediscovered, e.g. succinic acid, isosorbide, HMF (hydroxymethylfurfural), vegetal oils and glycerol. Recently, several research programs have been set up to stimulate the development of bio-based chemical products. ${ }^{1}$ One of these initiatives has led to the industrial production of isosorbide, a bicyclic diol derived from sorbitol, that has superior heat-resistant properties. Isosorbide diesters can be used as good substitutes for phthalate plasticizers, as exemplified by Polysorb ${ }^{\circledR}$ ID 37. As phthalates are increasingly under pressure because of their estrogenic and thus endocrine disrupting properties, the evaluation of possible substitutes is of major importance for the polymer industry since they still play a major role as plasticizer, next to adipates, citrates, epoxidized soybean oil and higher alcohols. ${ }^{2}$ Isosorbide modified with natural cinnamic acids can also be applied as a UV-absorbing plasticizer in sunscreens. ${ }^{3}$ 
The vegetable sources for the synthesis of renewable chemicals can still be divided in two classes, the food and non-food crops. As the non-food crops do not interfere with the food supply chain they can be used for non-food applications without any ethical concern. Castor oil, from the seeds of Ricinus communis (Euphorbiaceae) is an example of a non-food resource that is rich in ricinoleic acid (Figure 1, A). This unique C18 fatty acid with a hydroxyl group at position 12 and a double bond at position 9, can be easily converted in 10-undecylenic acid (further called UA) through pyrolysis. The literature on this unique fatty acid has recently been reviewed. ${ }^{4}$ Our research is concerned with the reactivity study of the fully renewable acyloin condensation product, that can be obtained through a sodium promoted coupling process of UA. ${ }^{5}$ In this manuscript, the reaction of this secondary alcohol with isocyanates is reported to obtain new urethanes with potential plasticizing or viscosity-modifying properties.

Several patents describe the reaction of various alcohols with mono- and/or diisocyanates for the plasticizing and rheological properties of the obtained compounds. For example $N$ octadecylbutylurethane (Figure 1, B) and analogues with the butyl group replaced by a C6 up to C22 linear alkyl chain are used in hot melt ink jet inks as viscosity controlling compounds. ${ }^{6} \mathrm{~N}$ Octadecylbutylurethane is used as an internal plasticizer in propellant compositions, successfully replacing dibutylphtalate, dioctylphtalate and triacetin. ${ }^{7}$ Analoguous compounds derived from diisocyanates and $\mathrm{C} 1$ up to $\mathrm{C} 22$ alcohols are used as carriers comprising $90-95 \%$ of ink jet inks ${ }^{8}$ or to prevent precipitation in pigment dispersions due to an increased stability. ${ }^{9}$

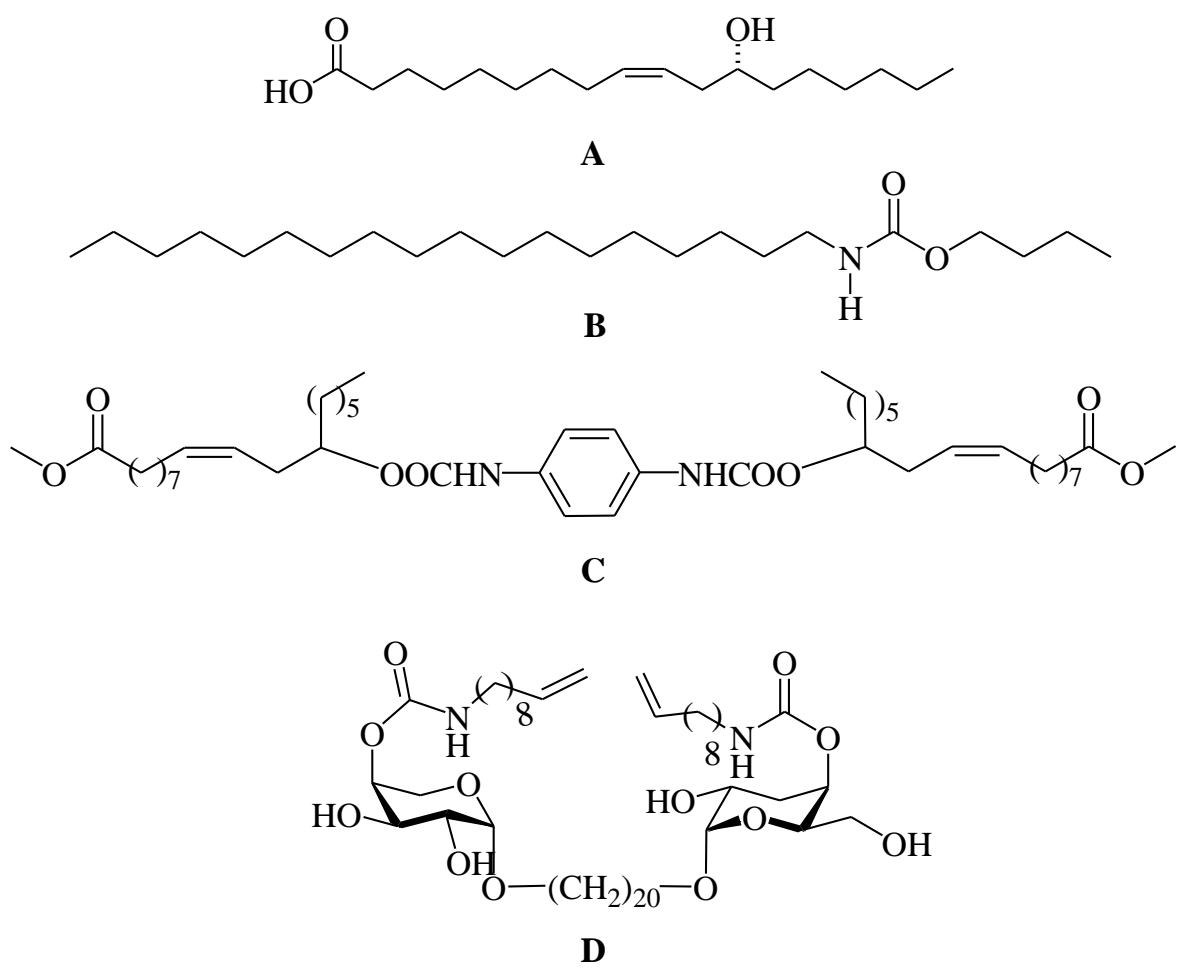

Figure 1. A: Ricinoleic acid, B-D: Important structures as functional material in plasticizers and as bolaforms. 
PVC-plasticizers were prepared from aromatic diisocyanates and methyl ricinoleate from castor oil (Figure 1, C) or a saturated analogue thereof. ${ }^{10}$

Besides plasticizers, the urethane function was also used to construct bolaforms from UA (Figure $1, \mathbf{D})$, which can be further converted into macrocyclic bolaforms via olefin metathesis. ${ }^{11}$

In our search towards new renewable building blocks from castor oil, the C22 acyloin derived from undecenoic acid was functionalized with several isocyanates towards new urethanes. During the experiments, the formation of 2-oxazolidinones was encountered in some cases. These ring-closed hemi-aminals could be good monomers for the production of polyurethanes through ring-opening polymerization as proven by the work of Höcker et al. ${ }^{12}$ Although some initial work has been done on the condensation of acyloins and benzoins with isocyanates, no reactions with aliphatic acyloins containing more than five carbon atoms have ever been reported before.

\section{Results and Discussion}

The first part of this work deals with the addition of acyloin 1 (10\% solution) to monofunctional isocyanates with the formation of compounds 2 (Scheme 1 and Table 1). Although the reaction was expected to be very straightforward, the experiments proved to be much more complicated in terms of obtaining an acceptable conversion.

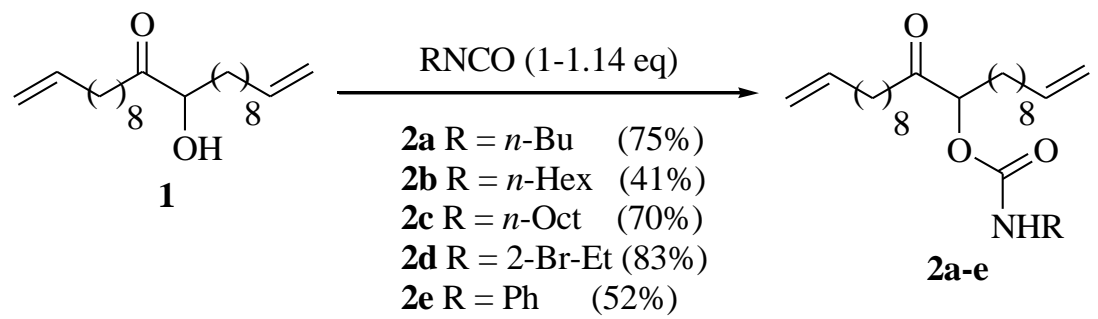

Scheme 1. Nucleophilic addition of acyloin 1 to monofunctional isocyanates.

Table 1. Reaction parameters for addition of monofunctional isocyanates to acyloin $\mathbf{1}$

\begin{tabular}{ccccccc}
\hline Compd & $\mathrm{R}$ & $\begin{array}{c}\text { Equiv } \\
\mathrm{RNCO}\end{array}$ & Solvent & $\begin{array}{c}\text { Temp } \\
\left({ }^{\circ} \mathrm{C}\right)\end{array}$ & $\begin{array}{c}\text { Time } \\
(\mathrm{h})\end{array}$ & $\begin{array}{c}\text { Isolated yield } \\
(\%)\end{array}$ \\
\hline $\mathbf{2 a}$ & $n$ - $\mathrm{Bu}$ & 1.14 & toluene & 92 & 3 & $75^{\mathrm{a}}$ \\
$\mathbf{2 b}$ & $n$-Hex & 1.05 & $1,2-\mathrm{C}_{2} \mathrm{H}_{4} \mathrm{Cl}_{2}$ & 83 & 3 & $41^{\mathrm{a}}$ \\
$\mathbf{2 c}$ & $n$-Oct & 1.00 & $1,2-\mathrm{C}_{2} \mathrm{H}_{4} \mathrm{Cl}_{2}$ & 83 & 4 & $70^{\mathrm{b}}$ \\
$\mathbf{2 d}$ & $2-\mathrm{Br}-\mathrm{Et}$ & 1.00 & toluene & 110 & 16 & $83^{\mathrm{a}}$ \\
$\mathbf{2 e}$ & $\mathrm{Ph}$ & 1.14 & toluene & 89 & 4 & $52^{\mathrm{a}}$ \\
\hline
\end{tabular}

${ }^{a}$ purified on silica column using a 15:1 or 10:1 (for 2d) petroleum ether:ethyl acetate mixture.

${ }^{\mathrm{b}}$ recrystallized from petroleum ether. 
First, the reaction was conducted with $n$-butyl isocyanate in dichloromethane, but was not successful at room temperature nor at reflux temperature for $24 \mathrm{~h}$. Higher boiling solvents, such as tetrahydrofuran and toluene, converted the acyloin almost completely after three hours at reflux temperature and at $92^{\circ} \mathrm{C}$, respectively. This indicated that a temperature range of $65-92^{\circ} \mathrm{C}$ was appropriate to perform this reaction. 1,2-Dichloroethane (boiling point of $83^{\circ} \mathrm{C}$ ) was therefore also evaluated as a solvent for this reaction (for compound $\mathbf{2 b}, \mathbf{2 c}, \mathbf{3}$ and $\mathbf{4}$ (see further)). When higher temperatures were applied, side reactions took place for compound $\mathbf{2 a}$ (vide infra). The reaction with 2-bromoethyl isocyanate required refluxing in toluene for $16 \mathrm{~h}$ to reach $95 \%$ conversion. Prolonged heating at reflux $(72 \mathrm{~h})$ to further increase the conversion, resulted in the formation of two products in a 1:1 molar ratio (H-NMR spectrum) and a dibrominated product that was visible in the mass spectrum. Further purification in order to characterize these products was however not successful. Compounds 2a, 2c and 2d were obtained in moderate to good yields. For compound $\mathbf{2 b}$, on the contrary, only $41 \%$ was isolated after column chromatography. A possible explanation is the rather low melting point $\left(30^{\circ} \mathrm{C}\right)$ that may cause some losses during column chromatography at room temperature and which makes recrystallization rather difficult to perform. The amount of isocyanate used in the reactions was approximately one equivalent (as optimized by performing experiments with varying amounts of equivalents) and is given in Table 1.

The observations for the reaction with phenyl isocyanate differ from these with acyclic, aliphatic isocyanates already mentioned. More polar compounds eluted from the column (using pure ethyl acetate) after compound $\mathbf{2 e}$ had eluted, and were isolated in $39 \%$ yield. After further chromatographical purification, a white, solid compound was found to be the 5-membered ring closed hemiaminal derivative $\mathbf{2 f}$ (Figure 2), formed by nucleophilic attack of the urethane nitrogen onto the carbonyl function.

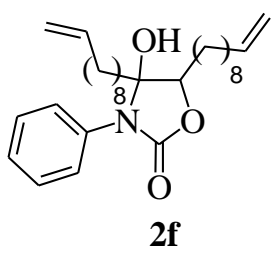

Figure 2. Ring closed hemiaminal $\mathbf{2 f}$ formed from urethane 2e during column chromatography.

Compound $2 \mathrm{f}$ was mainly formed on the silica column since it was hardly visible in the spectrum of the original reaction mixture. The structure of this compound was confirmed by comparison of the spectral data with published data on short chain analogous hemiaminals. Especially the reported ppm-values of the oxazolidinone ring in ${ }^{1} \mathrm{H}$ - and ${ }^{13} \mathrm{C}$-NMR were in accordance with the observed values for compound $\mathbf{2 f}$ (Table 2). ${ }^{13,14}$ The presence of a peak with the molecular weight of the dehydrated product in the mass spectrum was also observed to be characteristic as well as the aromatic multiplet in the ${ }^{1} \mathrm{H}-\mathrm{NMR}$ comprising all the aromatic proton signals (and no separate peak for the para protons). ${ }^{14}$ This ring closure was also observed 
for compound $2 \mathrm{a}$ when it was heated for $1.5 \mathrm{~h}$ at $92^{\circ} \mathrm{c}$ followed by heating at $100^{\circ} \mathrm{C}$. After chromatography, $62 \%$ of the isolated products proved to be the oxazolidinone derivative as confirmed by ${ }^{1} \mathrm{H}-\mathrm{NMR}$, although it was not completely pure. The reaction temperature is therefore a critical factor. As for compounds $\mathbf{2 b - 2 d}$ no such ring closure was observed at the reported reaction conditions.

Table 2. Comparison of ppm-values obtained for compound $\mathbf{2 f}$ with literature ${ }^{13,14}$

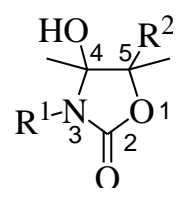

\begin{tabular}{|c|c|c|c|c|c|}
\hline \multicolumn{2}{|c|}{ Compd } & $2 f$ & $\begin{array}{c}\mathrm{R}^{1}=\mathrm{Ph} \\
\mathrm{R}^{2}=\mathrm{H}^{13}\end{array}$ & $\begin{array}{c}\mathrm{R}^{1}=p-\mathrm{CH}_{3} \mathrm{Ph} \\
\mathrm{R}^{2}=\mathrm{Me}^{14}\end{array}$ & $\begin{array}{c}\mathrm{R}^{1}=\mathrm{PhCH}_{2} \\
\mathrm{R}^{2}=\mathrm{Me}^{14}\end{array}$ \\
\hline \multirow{3}{*}{$\begin{array}{c}{ }^{1} \mathrm{H} \text {-signals } \\
(\mathrm{ppm})\end{array}$} & $\mathrm{C} 5-\underline{\mathrm{H}}$ & $4.27-4.31$ & 4.42 & - & - \\
\hline & $\mathrm{C} 4-\mathrm{OH}$ & 4.79 & 4.98 & 4.62 & 4.18 \\
\hline & $\mathrm{Ph}$ & $7.23-7.43$ & $7.25-7.50$ & - & - \\
\hline \multirow{3}{*}{$\begin{array}{c}{ }^{13} \mathrm{C} \text {-signals } \\
\text { (ppm) }\end{array}$} & $\mathrm{C} 5$ & 82.9 & 80.9 & - & - \\
\hline & $\mathrm{C} 4$ & 91.1 & 89.2 & - & - \\
\hline & $\mathrm{C} 2$ & 157.0 & 156.8 & - & - \\
\hline
\end{tabular}

The formation of 4-oxazolin-2-ones has been observed from low molecular weight acyloins and benzoins in DMF/pyridine. Only the ring-closed product and not the open carbamoylated benzoin could be isolated (with low yield). ${ }^{15}$ The reaction of 3-Hydroxy-3-methyl-2-butanone with aromatic isocyanates leads to cyclic 4-hydroxy-2-oxazolidinones that were dehydrated to exocyclic 4-methylen-2-oxazolidinones. ${ }^{16,17}$ More recent publications show that this condensation towards open chain carbamates or cyclic hemiaminals (anti and syn adducts) and dehydrated exo- and endo-adducts is thermodynamically controlled. ${ }^{13,18}$

The addition of bifunctional isocyanates (hexamethylene diisocyanate and toluene 2,4diisocyanate) has also been evaluated (Scheme 2). For the aliphatic diisocyanate, the reaction was complete after 5 hours of reflux in 1,2-dichloroethane. Recrystallization from petroleum ether yielded $86 \%$ of compound 3 . The formation of ring closed derivatives was not observed in this case, which can be explained by the sterical hindrance in urethane 3 . Condensation of acyloin 1 with toluene-2,4-diisocyanate in the same solvent for 3.5 hours, seemed successful judging the NMR spectra. During column chromatography, however, the NMR-spectra of successive collected fractions started to show deviations from the original spectrum. Comparison with the spectrum of $\mathbf{2 f}$, revealed some characteristics of the ring closed side product and, judging from the integrations in the ${ }^{1} \mathrm{H}-\mathrm{NMR}$, ring closure occurred only at one side of the aromatic ring (61\%), although no clear conclusions could be drawn from the mass spectrum and 
the ${ }^{1} \mathrm{H}-\mathrm{NMR}$-spectrum was not completely pure. Nevertheless, it was possible to isolate product 4 in $22 \%$ yield in the first fractions of the chromatographical separation.

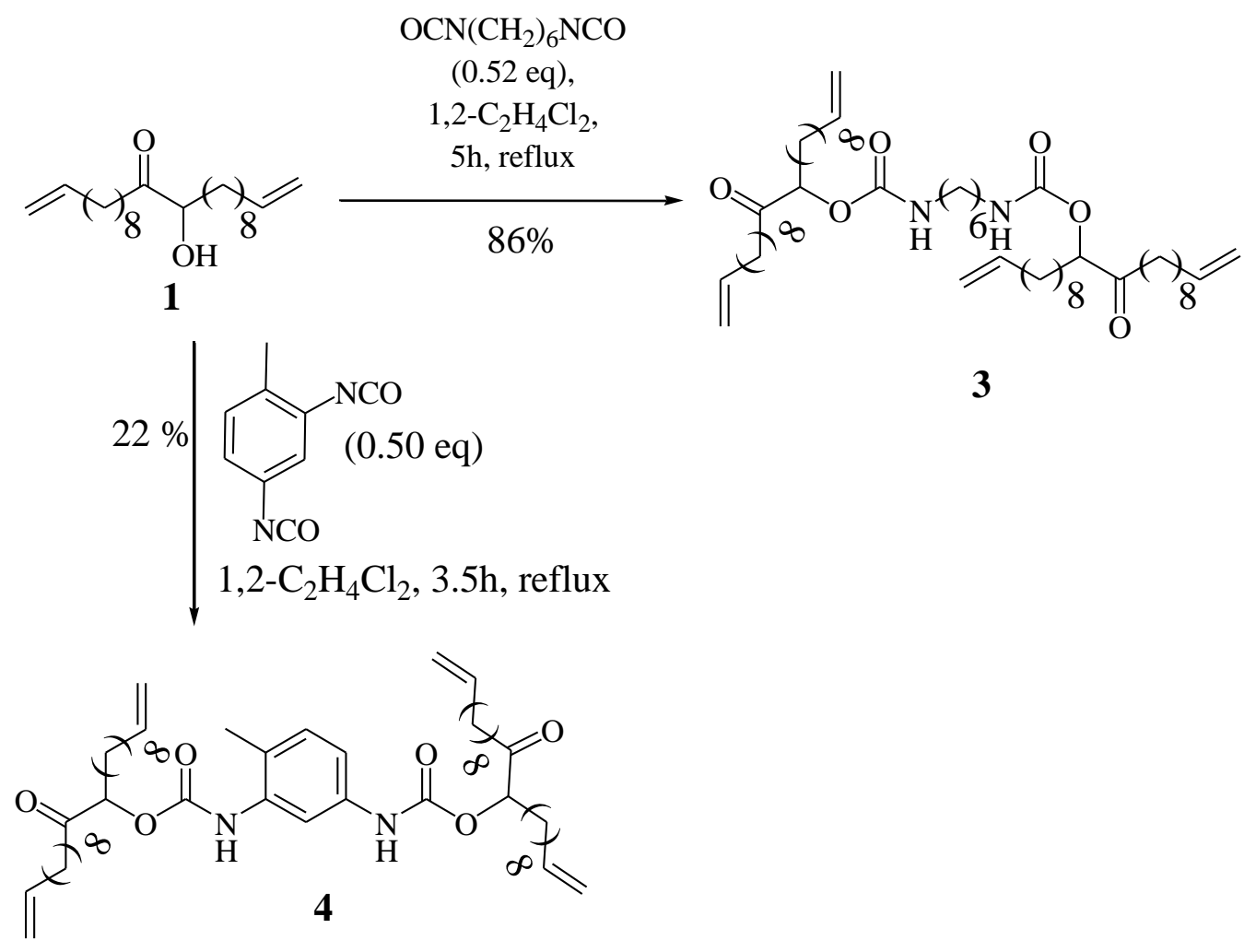

Scheme 2. Nucleophilic addition of bifunctional isocyanates to acyloin $\mathbf{1}$ (indicated yields are isolated yields).

\section{Conclusions}

In summary, novel aliphatic and aromatic urethane derivatives of a renewable C22-acyloin could be prepared in moderate yield. In most cases only the open chain urethane was isolated, but in the case of the aromatic and the $n$-butyl derivatives, the ring closed hemiaminal was also isolated. The formation of these compounds occurred mainly during column chromatography (for the aromatic derivatives) or at higher reaction temperatures (for the n-butyl derivative). These urethanes derived from renewable undecenoic acid may be used as additives for the polymer industry. Their properties are being evaluated and will be reported in due course. 


\section{Experimental Section}

General. High-resolution ${ }^{1} \mathrm{H}$ NMR (300 MHz) and ${ }^{13} \mathrm{C}$ NMR (75 MHz) spectra were obtained with a Jeol JNM-EX 300 NMR spectrometer. Peak assignments were confirmed using DEPT and 2D-HSQC, HMBC and COSY spectra. MS spectra were recorded on an Agilent 1100 Series VS $(\mathrm{ES}, 4000 \mathrm{~V})$ mass spectrometer. High Resolution mass spectra for compounds $\mathbf{2 a}, \mathbf{2 e}$ and $\mathbf{3}$ were determined on a Finnigan MAT95XP Tandem Mass Spectrometer by Positive Electrospray Ionization (+ESI) with direct injection in the sample loop and without separation of compounds. The mobile phase consisted of 50/50 acetonitrile/water $+0.1 \%$ formic acid $\left(50 \mu \mathrm{lmin}^{-1}\right)$. The vaporization took place at room temperature and a heated capillary of $250{ }^{\circ} \mathrm{C}$ was used. A corona current of $2 \mu \mathrm{A}$ was applied and a mass resolution of 8000 was obtained. The sheath gas was $\mathrm{N}_{2}$ (4 bar). Internal reference ions were PEG and PPG ions. The other compounds (2b, 2c, 2d, 2f and 4), were analyzed on an Agilent 6220 Accurate-Mass TOF LC/MS equipped with APCI/ESI multimode source. Samples were recorded using direct infusion and $4 \mathrm{GHz}$ High Resolution Mode. IR-spectra were obtained with a Perkin Elmer Spectrum BX apparatus (ATRmeasurement). Melting points of crystalline compounds were measured with a Büchi 540 apparatus and are uncorrected. Rf values were calculated from glass plates coated with a thin layer of silicagel $60 \mathrm{~F}_{254}$ (Merck). All reagents were purchased from Sigma-Aldrich or from Acros Organics and were used as such unless otherwise stated. The acyloin (12-hydroxydocosa1,21-dien-11-one) was prepared following the procedure described. ${ }^{14} \mathrm{~A}$ more straightforward procedure for the crystallization of the acyloin from petroleum ether at $-18{ }^{\circ} \mathrm{C}$ (in stead of from boiling dichloromethane and methanol), was however used. All reactions were performed using a Schlenck apparatus to keep the reagents under nitrogen gas and exclude water from the reaction medium. All solvents used were dried by distillation after the addition of sodium and benzophenone as an indicator or were kept on molecular sieves to remove water.

\section{General procedure for the reaction of acyloin 1 with monofunctional isocyanates (see Table} 1 for solvents, equivalents of isocyanate used, reaction temperatures and times and purification methods)

To a flame dried 50mL flask a 10\%-solution of acyloin $\mathbf{1}$ in extra dry solvent was added and kept under a nitrogen atmosphere. For compounds $\mathbf{2 a}$ and $\mathbf{2 e}, 1.61 \mathrm{~g}$ acyloin $(4.8 \mathrm{mmol})$ was used, for compound $\mathbf{2 b}, 1.29 \mathrm{~g}(3.8 \mathrm{mmol})$, for compound $\mathbf{2 c}, 1.35 \mathrm{~g}(4.0 \mathrm{mmol})$ and for compound $\mathbf{2 d}$, $3.36 \mathrm{~g}(0.01 \mathrm{~mol})$. Then, isocyanate was added volumetrically through a septum using a syringe. The reaction mixture was heated at the mentioned temperature and time. ${ }^{1} \mathrm{H}$ NMR of the mixture revealed a conversion of $90 \%$ or more. The solvent was evaporated and the mixture was purified by column chromatography on silica gel using petroleum ether/ethyl acetate (compounds $\mathbf{2 a}, \mathbf{2 b}$, $\mathbf{2 d}, \mathbf{2 e}$ and $\mathbf{2 f}$ ) or was recrystallized from petroleum ether (2c).

(12-Oxodocosa-1,21-dien-11-yl) $\boldsymbol{n}$-butylcarbamate (2a). Colorless oil, yield 75\%, 1.56 g. ${ }^{1} \mathrm{H}$ NMR $\left(300 \mathrm{MHz}, \mathrm{CDCl}_{3}\right): \delta_{\mathrm{H}} 0.90-0.95\left(\mathrm{t},{ }^{3} J_{\mathrm{H}, \mathrm{H}}=7.5 \mathrm{~Hz}, 3 \mathrm{H}, \mathrm{C}_{3}\right), 1.27-1.74\left(\mathrm{~m}, 30 \mathrm{H}, \underline{\mathrm{C}}_{2}\right)$, 2.00-2.07 (m, $4 \mathrm{H}, \mathrm{CH}=\mathrm{CH}_{2}-\mathrm{C}_{2}$ ), 2.34-2.55 (two times dxt, ${ }^{2} J_{\mathrm{Ha}, \mathrm{Hb}}=17.6 \mathrm{~Hz},{ }^{3} \mathrm{~J}_{\mathrm{Ha}, \mathrm{H}}=7.4 \mathrm{~Hz}$, 
$\left.{ }^{3} J_{\mathrm{Hb}, \mathrm{H}}=7.3 \mathrm{~Hz}, 2 \mathrm{H}, \underline{\mathrm{C}}_{2} \mathrm{CO}\right), 3.15-3.22\left(\mathrm{~m}, 2 \mathrm{H}, \underline{\mathrm{C}}_{2}-\mathrm{NH}\right), 4.81-4.85$ broad triplet, ${ }^{3} J_{\mathrm{H}, \mathrm{H}}=5.7$ $\mathrm{Hz}, 1 \mathrm{H}, \mathrm{NH}), 4.91-5.02\left(\mathrm{~m}, 5 \mathrm{H},-\mathrm{C} \underline{\mathrm{HOCO}}+\mathrm{C}_{\underline{\mathrm{H}}}=\mathrm{CH}-\right), 5.74-5.88\left(\mathrm{dxdxt},{ }^{3} \mathrm{~J}_{\mathrm{H}, \mathrm{H}}=6.6 \mathrm{~Hz}\right.$, $\left.{ }^{3} J_{\mathrm{H}, \mathrm{Hcis}}=10.4 \mathrm{~Hz},{ }^{3} J_{\mathrm{H}, \mathrm{Htrans}}=17.1 \mathrm{~Hz}, 2 \mathrm{H}, \mathrm{CH}_{2}=\mathrm{CH}-\right) .{ }^{13} \mathrm{C} \mathrm{NMR}\left(75.6 \mathrm{MHz}, \mathrm{CDCl}_{3}\right): \delta_{\mathrm{C}} 13.8$ $\left(\underline{\mathrm{CH}}_{3}\right), 19.9,23.2,25.3,29.0,29.1,29.2,29.4,29.8,30.8,32.0\left(\underline{\mathrm{CH}}_{2}\right), 33.8\left(\mathrm{CH}_{2}=\mathrm{CH}-\underline{\mathrm{CH}}_{2}\right), 38.7$ $\left(\underline{\mathrm{CH}}_{2}-\mathrm{CO}\right), 40.9\left(\underline{\mathrm{CH}}_{2}-\mathrm{NH}\right), 78.5(\underline{\mathrm{C}} \mathrm{HOCO}), 114.2\left(\underline{\mathrm{CH}}_{2}=\mathrm{CH}\right), 139.2 \quad\left(\mathrm{CH}_{2}=\underline{\mathrm{CH}}\right), 156.0$ (OCONHR), 209.1 ( $\underline{\mathrm{CO}}$ ). IR (ATR, $v, \mathrm{~cm}^{-1}$ ): 3356 (broad, w, NH), 3076 (w, $\mathrm{CH}_{2}=\mathrm{CH}$ ), 2925 ( $v_{\max }$ alkyl), 2854 (s, alkyl), $1716(\mathrm{~s}, \mathrm{CO}), 1640$ (w, $\left.\mathrm{CH}_{2}=\mathrm{CH}\right) . \mathrm{MS}(+\mathrm{ESI}, 4000 \mathrm{~V}): \mathrm{m} / \mathrm{z}(\%)=$ $436(\mathrm{M}+1,100)$. HRMS (+ESI) calcd for $\mathrm{C}_{27} \mathrm{H}_{50} \mathrm{NO}_{3}\left(\mathrm{M}^{+}+1\right)$ : 436.37852 ; found: 436.37937.

4,5-Bis(dec-9-enyl)-4-hydroxy-3-n-butyloxazolidin-2-one. Yield 62\%, 0.81 g. ${ }^{1} \mathrm{H}$ NMR (300 $\left.\mathrm{MHz}, \mathrm{CDCl}_{3}\right): \delta_{\mathrm{H}} 0.8-1.0\left(\mathrm{~m}, 3 \mathrm{H}, \mathrm{C}_{3}\right), 1.1-1.8\left(\mathrm{~m}, 32 \mathrm{H}, \underline{\mathrm{CH}_{2}}\right), 2.0-2.1\left(\mathrm{~m}, 4 \mathrm{H}, \mathrm{CH}=\mathrm{CH}_{2}-\right.$ $\left.\mathrm{C}_{2}\right)$, 3.0-3.3 (m, $\left.2 \mathrm{H}, \mathrm{CH}_{2} \mathrm{~N}\right), 4.1-4.2\left(\mathrm{~m}, 1 \mathrm{H}, \mathrm{C}_{\text {oxazolidinone }}\right.$ ), $4.9-5.1$ (m, $\left.4 \mathrm{H}, \underline{\mathrm{C}}_{2}=\mathrm{CH}-\right)$, 5.7$5.9\left(\mathrm{~m}, 2 \mathrm{H}, \mathrm{CH}_{2}=\mathrm{CH}-\right.$ ).

(12-Oxodocosa-1,21-dien-11-yl) $\boldsymbol{n}$-hexylcarbamate (2b). White waxy material, yield $41 \%$, $0.73 \mathrm{~g}, \mathrm{mp}: 30{ }^{\circ} \mathrm{C} .{ }^{1} \mathrm{H}$ NMR $\left(300 \mathrm{MHz}, \mathrm{CDCl}_{3}\right): \delta_{\mathrm{H}} 0.86-0.91\left(\mathrm{t},{ }^{3} J_{\mathrm{H}, \mathrm{H}}=6.9 \mathrm{~Hz}, 3 \mathrm{H}, \mathrm{C}_{3}\right), 1.24$ - 1.74 (m, 34H, $\mathrm{CH}_{2}$ ), 2.00-2.07 (m, $4 \mathrm{H}, \mathrm{CH}=\mathrm{CH}_{2}-\mathrm{C}_{2}$ ), 2.34-2.55 (two times dxt, ${ }^{2} J_{\mathrm{Ha}, \mathrm{Hb}}=17.6$ $\left.\mathrm{Hz},{ }^{3} \mathrm{Ja}_{\mathrm{Ha}, \mathrm{H}}=7.4 \mathrm{~Hz},{ }^{3} \mathrm{~J}_{\mathrm{Hb}, \mathrm{H}}=7.4 \mathrm{~Hz}, 2 \mathrm{H}, \mathrm{C}_{2} \mathrm{CO}\right), 3.14-3.21\left(\mathrm{~m}, 2 \mathrm{H}, \mathrm{C}_{2}-\mathrm{NH}\right), 4.81-4.85$ (broad triplet, $\left.{ }^{3} J_{\mathrm{H}, \mathrm{H}}=5.7 \mathrm{~Hz}, 1 \mathrm{H}, \mathrm{NH}\right), 4.91-5.02\left(\mathrm{~m}, 5 \mathrm{H},-\mathrm{C} \underline{\mathrm{HOCO}}+\mathrm{C}_{2}=\mathrm{CH}-\right), 5.74-5.88(\mathrm{dxdxt}$, $\left.{ }^{3} J_{\mathrm{H}, \mathrm{H}}=6.6 \mathrm{~Hz},{ }^{3} J_{\mathrm{H}, \mathrm{H} \text { cis }}=10.4 \mathrm{~Hz},{ }^{3} J_{\mathrm{H}, \mathrm{H} \text { trans }}=17.1 \mathrm{~Hz}, 2 \mathrm{H}, \mathrm{CH}_{2}=\mathrm{CH}-\right) .{ }^{13} \mathrm{C}$ NMR $(75.6 \mathrm{MHz}$, $\left.\mathrm{CDCl}_{3}\right): \delta_{\mathrm{C}} 14.0\left(\underline{\mathrm{CH}}_{3}\right), 22.6,23.1,25.3,26.4,28.9,29.1,29.2,29.3,29.9,30.7,31.5\left(\underline{\mathrm{CH}}_{2}\right), 33.8$ $\left(\mathrm{CH}_{2}=\mathrm{CH}-\underline{\mathrm{CH}}_{2}\right), 38.6\left(\underline{\mathrm{CH}}_{2}-\mathrm{CO}\right), 41.1\left(\underline{\mathrm{CH}}_{2}-\mathrm{NH}\right), 78.5(\underline{\mathrm{CHOCO}}), 114.2\left(\underline{\mathrm{CH}}_{2}=\mathrm{CH}\right), 139.2$


$\mathrm{CH}_{2}=\mathrm{CH}$ ), 2922 ( $v_{\max }$, alkyl), 2852 (s, alkyl), 1712 (w, CO), 1693 (s, CO), 1642 (w, $\mathrm{CH}_{2}=\mathrm{CH}$ ). MS (+ESI, $4000 \mathrm{~V}): m / z(\%)=464(\mathrm{M}+1,100)$. HRMS (+ESI) calcd for $\mathrm{C}_{29} \mathrm{H}_{54} \mathrm{NO}_{3}\left(\mathrm{M}^{+}+1\right)$ : 464.4098; found: 464.4098 .

(12-Oxodocosa-1,21-dien-11-yl) $\boldsymbol{n}$-octylcarbamate (2c). White powder, yield 70\%, $1.38 \mathrm{~g}$, mp: 45-46 ${ }^{\circ} \mathrm{C} .{ }^{1} \mathrm{H} \mathrm{NMR}\left(300 \mathrm{MHz}, \mathrm{CDCl}_{3}\right): \delta_{\mathrm{H}} 0.86-0.90\left(\mathrm{t},{ }^{3} \mathrm{~J}_{\mathrm{H}, \mathrm{H}}=6.4 \mathrm{~Hz}, 3 \mathrm{H}, \mathrm{C}_{3}\right), 1.27-1.71(\mathrm{~m}$, $38 \mathrm{H}, \mathrm{C}_{2}$ ) , 2.00-2.07 (m, 4H, CH= $\mathrm{CH}_{2}-\mathrm{CH}_{2}$ ), 2.34-2.55 (two times dxt, ${ }^{2} J_{\mathrm{Ha}, \mathrm{Hb}}=17.6 \mathrm{~Hz},{ }^{3} \mathrm{~J}_{\mathrm{Ha}, \mathrm{H}}$ $\left.=7.4 \mathrm{~Hz},{ }^{3} J_{\mathrm{Hb}, \mathrm{H}}=7.4 \mathrm{~Hz}, 2 \mathrm{H}, \underline{\mathrm{C}}_{2} \mathrm{CO}\right), 3.14-3.20\left(\mathrm{~m}, 2 \mathrm{H}, \mathrm{C}_{2}-\mathrm{NH}\right), 4.88-5.01(\mathrm{~m}, 6 \mathrm{H},-$ $\left.\mathrm{C} \underline{\mathrm{HOCO}}+\mathrm{C}_{\underline{\mathrm{H}}}=\mathrm{CH}-+\mathrm{NH}\right), 5.74-5.87\left(\mathrm{dxdxt},{ }^{3} J_{\mathrm{H}, \mathrm{H}}=6.6 \mathrm{~Hz},{ }^{3} J_{\mathrm{H}, \mathrm{H}}\right.$ cis $=10.4 \mathrm{~Hz}, J_{\mathrm{H}, \mathrm{H}}$ trans $=17.1$ $\left.\mathrm{Hz}, 2 \mathrm{H}, \mathrm{CH}_{2}=\mathrm{C} \underline{\mathrm{H}}-\right) .{ }^{13} \mathrm{C} \mathrm{NMR}\left(75.6 \mathrm{MHz}, \mathrm{CDCl}_{3}\right): \delta_{\mathrm{C}} 14.2\left(\underline{\mathrm{CH}}_{3}\right), 22.7,23.2,25.3,26.8,29.0$, 29.2, 29.4, 30.0, 30.8, $31.9\left(\underline{\mathrm{CH}}_{2}\right), 33.9\left(\mathrm{CH}_{2}=\mathrm{CH}-\underline{\mathrm{CH}}_{2}\right), 38.7\left(\underline{\mathrm{CH}}_{2}-\mathrm{CO}\right), 41.2\left(\underline{\mathrm{CH}}_{2}-\mathrm{NH}\right), 78.6$


$\mathrm{cm}^{-1}$ ): 3302(sharp, m, $\mathrm{NH}$ ), 3080 (w, $\mathrm{CH}_{2}=\mathrm{CH}$ ), 2921 ( $v_{\max }$, alkyl), 2851 (s, alkyl), 1718 (w, $\mathrm{CO}), 1694$ (s, CO), 1642 (w, $\left.\mathrm{CH}_{2}=\mathrm{CH}\right)$. MS (+ESI, $\left.4000 \mathrm{~V}\right): \mathrm{m} / \mathrm{z}(\%)=492(\mathrm{M}+1,100)$. HRMS (+ESI) calcd for $\mathrm{C}_{31} \mathrm{H}_{58} \mathrm{NO}_{3}\left(\mathrm{M}^{+}+1\right)$ : 492.4411; found: 492.4436 .

(12-Oxodocosa-1,21-dien-11-yl) 2-bromoethylcarbamate (2d). Pale yellow waxy oil, yield 83\%, 4.01 g. ${ }^{1} \mathrm{H}$ NMR(300 MHz, $\mathrm{CDCl}_{3}$ ) $): \delta_{\mathrm{H}} 1.28-1.73\left(\mathrm{~m}, 26 \mathrm{H}, \mathrm{C}_{2}\right), 2.00-2.07(\mathrm{~m}, 4 \mathrm{H}$, $\mathrm{CH}=\mathrm{CH}_{2}-\mathrm{C}_{2}$ ), 2.32-2.55 (two times dxt, ${ }^{2} J_{\mathrm{Ha}, \mathrm{Hb}}=17.6 \mathrm{~Hz},{ }^{3} J_{\mathrm{Ha}, \mathrm{H}}=7.4 \mathrm{~Hz},{ }^{3} J_{\mathrm{Hb}, \mathrm{H}}=7.5 \mathrm{~Hz}, 2 \mathrm{H}$, C$\left.\underline{\mathrm{H}}_{2} \mathrm{CO}\right), 3.45-3.49\left(\mathrm{~m}, 2 \mathrm{H}, \underline{\mathrm{CH}}_{2}-\mathrm{Br}\right), 3.54-3.66\left(\mathrm{~m}, 2 \mathrm{H}, \underline{\mathrm{C}}_{2}-\mathrm{NH}\right), 4.91-5.02(\mathrm{~m}, \mathrm{H},-\mathrm{C} \underline{\mathrm{HOCO}}$ $\left.+\underline{\mathrm{C}}_{2}=\mathrm{CH}-\right), 5.35-5.39$ (broad triplet, $\left.{ }^{3} J_{\mathrm{H}, \mathrm{H}}=6.1 \mathrm{~Hz}, 1 \mathrm{H}, \mathrm{N} \underline{\mathrm{H}}\right), 5.74-5.87\left(\mathrm{dxdxt},{ }^{3} J_{\mathrm{H}, \mathrm{H}}=6.9 \mathrm{~Hz}\right.$, 
$\left.{ }^{3} J_{\mathrm{H}, \mathrm{H} \text { cis }}=10.2 \mathrm{~Hz},{ }^{3} J_{\mathrm{H}, \mathrm{H} \text { trans }}=17.1 \mathrm{~Hz}, 2 \mathrm{H}, \mathrm{CH}_{2}=\mathrm{CH}-\right) .{ }^{13} \mathrm{C} \mathrm{NMR}\left(75.6 \mathrm{MHz}, \mathrm{CDCl}_{3}\right): \delta_{\mathrm{C}} 23.2$, 25.3, 29.0, 29.1, 29.2, 29.4, 30.7, $32.3\left(\underline{\mathrm{CH}}_{2} \mathrm{Br}\right), 33.9\left(\mathrm{CH}_{2}=\mathrm{CH}-\underline{\mathrm{CH}}_{2}\right), 38.7\left(\underline{\mathrm{CH}}_{2}-\mathrm{CO}\right), 42.8$ $\left(\underline{\mathrm{C}} \mathrm{H}_{2}-\mathrm{NH}\right), 79.0(\underline{\mathrm{CHOCO}}), 114.3\left(\underline{\mathrm{CH}}_{2}=\mathrm{CH}\right), 139.2\left(\mathrm{CH}_{2}=\underline{\mathrm{CH}}\right), 155.8$ (OㅁNHR), $208.6(\underline{\mathrm{CO}})$. IR (ATR, $\left.v, \mathrm{~cm}^{-1}\right)$ : 3355 (broad, w, NH), 3075 (w, $\left.\mathrm{CH}_{2}=\mathrm{CH}\right), 2924$ ( $v_{\max }$ alkyl), 2854 (s, alkyl), 1717 (s, CO), 1640 (w, $\left.\mathrm{CH}_{2}=\mathrm{CH}\right), 1602\left(-\mathrm{C}_{6} \mathrm{H}_{5}\right)$. MS (+ESI, $\left.4000 \mathrm{~V}\right): \mathrm{m} / z(\%)=486(\mathrm{M}+1,66)$, 488 (M+3, 100). HRMS (+ESI) calcd for $\mathrm{C}_{25} \mathrm{H}_{45} \mathrm{BrNO}_{3}\left(\mathrm{M}^{+}+1\right)$ : 486.2577; found: 486.2575 .

(12-Oxodocosa-1,21-dien-11-yl) phenylcarbamate (2e). Yellow oil, yield 52\%, 1.14 g. ${ }^{1} \mathrm{H}$ $\operatorname{NMR}\left(300 \mathrm{MHz}, \mathrm{CDCl}_{3}\right): \delta_{\mathrm{H}} 1.26-1.83\left(\mathrm{~m}, 26 \mathrm{H}, \mathrm{CH}_{2}\right), 2.00-2.06\left(\mathrm{~m}, 4 \mathrm{H}, \mathrm{CH}=\mathrm{CH}_{2}-\mathrm{C}_{2}\right), 2.39-$ 2.59 (two times dxt, ${ }^{2} J_{\mathrm{Ha}, \mathrm{Hb}}=17.6 \mathrm{~Hz},{ }^{3} J_{\mathrm{Ha}, \mathrm{H}}=7.4 \mathrm{~Hz},{ }^{3} J_{\mathrm{Hb}, \mathrm{H}}=7.4 \mathrm{~Hz}, 2 \mathrm{H}, \mathrm{CH}_{2} \mathrm{CO}$ ), 3.15-3.22 $\left(\mathrm{m}, 2 \mathrm{H}, \underline{\mathrm{C}}_{2}-\mathrm{NH}\right), 4.91-5.07\left(\mathrm{~m}, 5 \mathrm{H},-\mathrm{C} \underline{\mathrm{HOCO}}+\mathrm{C}_{2}=\mathrm{CH}-\right), 5.74-5.87\left(\mathrm{dxdxt},{ }^{3} J_{\mathrm{H}, \mathrm{H}}=6.6 \mathrm{~Hz}\right.$, $\left.{ }^{3} J_{\mathrm{H}, \mathrm{H} \text { cis }}=10.5 \mathrm{~Hz},{ }^{3} J_{\mathrm{H}, \mathrm{H} \text { trans }}=17.1 \mathrm{~Hz}, 2 \mathrm{H}, \mathrm{CH}_{2}=\mathrm{CH}-\right), 6.85(\mathrm{~s}, 1 \mathrm{H}, \mathrm{N} \underline{\mathrm{H}}), 7.04-7.10(\mathrm{~m}, 1 \mathrm{H}, \mathrm{p}-$ $\left.\mathrm{C}_{6} \mathrm{H}_{5}\right)$, 7.26-7.39 (m, 4H, o,m- $\left.\mathrm{C}_{6} \mathrm{H}_{5}\right) .{ }^{13} \mathrm{C} \mathrm{NMR}\left(75.6 \mathrm{MHz}, \mathrm{CDCl}_{3}\right): \delta_{\mathrm{C}} 23.2,25.4,29.0,29.16$, 29.25, 29.39, 29.43, 29.8, 30.7 ( $\left.\underline{\mathrm{CH}}_{2}\right), 33.9\left(\mathrm{CH}_{2}=\mathrm{CH}-\underline{\mathrm{CH}}_{2}\right), 38.8\left(\underline{\mathrm{CH}}_{2}-\mathrm{CO}\right), 79.0$ (ㄸHOCO), 114.2, $114.3\left(\underline{\mathrm{CH}}_{2}=\mathrm{CH}\right), 118.8 \quad\left(\mathrm{~m}-\mathrm{C}_{6} \mathrm{H}_{5}\right), 123.8\left(\mathrm{p}-\mathrm{C}_{6} \mathrm{H}_{5}\right), 129.2\left(\mathrm{o}-\mathrm{C}_{6} \mathrm{H}_{5}\right), 137.6\left(\mathrm{C}_{\text {arom.quat. }}\right)$ 139.25, $139.28\left(\mathrm{CH}_{2}=\underline{\mathrm{CH}}\right), 152.9$ (OCONHPh), $208.4(\underline{\mathrm{CO}})$. IR (ATR, $\left.v, \mathrm{~cm}^{-1}\right): 3329$ (broad, w, $\mathrm{NH}), 3074$ (w, $\left.\mathrm{CH}_{2}=\mathrm{CH}\right), 2924$ ( $v_{\max }$, alkyl), 2854 (s, alkyl), 1711 (s, CO), 1640 (w, $\mathrm{CH}_{2}=\mathrm{CH}$ ), $1602\left(\mathrm{~m}, \mathrm{C}_{6} \mathrm{H}_{5}\right)$. MS $(+\mathrm{ESI}, 4000 \mathrm{~V}): \mathrm{m} / \mathrm{z}(\%)=456(\mathrm{M}+1,100)$. HRMS (+ESI) calcd for $\mathrm{C}_{29} \mathrm{H}_{46} \mathrm{NO}_{3}\left(\mathrm{M}^{+}+1\right)$ : 456.34722 ; found: 456.34754 .

4,5-Bis(dec-9-enyl)-4-hydroxy-3-phenyloxazolidin-2-one (2f). White solid, yield 39\%, $0.86 \mathrm{~g}$, mp 66-68 ${ }^{\circ} \mathrm{C} .{ }^{1} \mathrm{H}$ NMR(300 MHz, $\left.\mathrm{CDCl}_{3}\right): \delta_{\mathrm{H}} 1.11-1.39\left(\mathrm{~m}, 24 \mathrm{H}, \mathrm{C}_{2}\right), 1.49-1.74(\mathrm{~m}, 4 \mathrm{H}$,

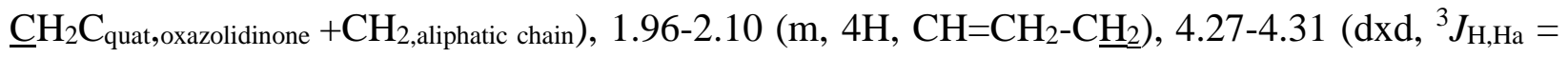
$\left.10.0 \mathrm{~Hz},{ }^{3} \mathrm{~J}_{\mathrm{H}, \mathrm{Hb}}=2.8 \mathrm{~Hz}, 1 \mathrm{H}, \mathrm{C}_{\text {oxazolidinone }}\right), 4.79(\mathrm{~s}, 1 \mathrm{H}, \mathrm{OH}), 4.90-5.04\left(\mathrm{~m}, 4 \mathrm{H}, \mathrm{C}_{2}=\mathrm{CH}-\right)$, 5.71-5.90 (m, $\left.2 \mathrm{H}, \mathrm{CH}_{2}=\mathrm{CH}-\right), 7.23-7.43\left(\mathrm{~m}, 5 \mathrm{H}, \mathrm{C}_{6} \mathrm{H}_{5}\right) .{ }^{13} \mathrm{C} \mathrm{NMR}\left(75.6 \mathrm{MHz}, \mathrm{CDCl}_{3}\right): \delta_{\mathrm{C}} 23.3$, 26.2, 28.9, 29.0, 29.17, 29.25, 29.48, 29.57( $\left.\underline{\mathrm{CH}}_{2}\right), \quad 33.8, \quad 33.9 \quad\left(\mathrm{CH}_{2}=\mathrm{CH}_{-} \mathrm{CH}_{2}\right), \quad 36.4$ $\left(\underline{\mathrm{C}} \mathrm{H}_{2} \mathrm{C}_{\text {quat, oxazolidinone }}\right), \quad 82.9$ ( $\left.\mathrm{CH}_{\text {oxazolidinone }}\right), 91.1 \quad\left(\mathrm{C}_{\text {quat, oxazolidinone }}\right), 114.3 \quad\left(\underline{\mathrm{C}} \mathrm{H}_{2}=\mathrm{CH}\right), 127.1$ $\left(\mathrm{C}_{6} \mathrm{H}_{5}\right), 127.2\left(\mathrm{p}-\mathrm{C}_{6} \mathrm{H}_{5}\right), 128.8\left(\mathrm{C}_{6} \mathrm{H}_{5}\right), 134.9$ ( $\left.\mathrm{C}_{\text {arom.quat. }}\right)$ 139.22, $139.28\left(\mathrm{CH}_{2}=\underline{\mathrm{C}} \mathrm{H}\right), 157.0$ (OCONHPh). IR (ATR, v, cm ${ }^{-1}$ ): 3314 (sharp, m, OH), 3076 (w, $\mathrm{CH}_{2}=\mathrm{CH}$ ), 2924 (s, alkyl), 2852 (m, alkyl), 1725 ( $\left.v_{\max }, \mathrm{OCONHPh}\right), 1640\left(\mathrm{w}, \mathrm{CH}_{2}=\mathrm{CH}\right), 1599$ (m, $\left.\mathrm{C}_{6} \mathrm{H}_{5}\right)$. MS (+ESI, $\left.4000 \mathrm{~V}\right)$ : $m / z(\%)=456(\mathrm{M}+1,89), 438\left(\mathrm{M}+1-\mathrm{H}_{2} \mathrm{O}, 100\right)$. HRMS $(+\mathrm{ESI})$ calcd for $\mathrm{C}_{29} \mathrm{H}_{46} \mathrm{NO}_{3}\left(\mathrm{M}^{+}+1\right)$ : 456.3472; found: 456.3473 .

General procedure for the reaction of acyloin 1 with bifunctional hexamethylene diisocyanate

To a flame dried $50 \mathrm{~mL}$ flask a solution of acyloin $(1.29 \mathrm{~g}, 3.8 \mathrm{mmol})$ in extra dry 1,2dichloroethane ( dried with molecular sieves, $15 \mathrm{~mL}$ ) was added and placed under nitrogen gas. Then hexamethylene diisocyanate $(0.336 \mathrm{~g}, 2 \mathrm{mmol}, 0.52 \mathrm{eq})$ was added by means of a syringe through a septum. The reaction mixture was refluxed for $5 \mathrm{~h}$. In ${ }^{1} \mathrm{H}$ NMR a conversion of more than $95 \%$ was observed. After evaporation of the solvent, product $\mathbf{3}$ was recrystallized from petroleum ether. 
Bis(12-oxodocosa-1,21-dien-11-yl) 1,6-hexanedicarbamate (3). White powder, yield 86\%, $1.39 \mathrm{~g}, \mathrm{mp} 51-54{ }^{\circ} \mathrm{C} .{ }^{1} \mathrm{H} \mathrm{NMR}\left(300 \mathrm{MHz}, \mathrm{CDCl}_{3}\right): \delta_{\mathrm{H}} 1.27-1.71\left(\mathrm{~m}, 60 \mathrm{H}, \mathrm{CH}_{2}\right), 2.00-2.07(\mathrm{~m}$, $8 \mathrm{H}, \mathrm{CH}=\mathrm{CH}_{2}-\underline{\mathrm{C}}_{2}$ ), 2.34-2.55 (two times dxt, ${ }^{2} J_{\mathrm{Ha}, \mathrm{Hb}}=17.6 \mathrm{~Hz},{ }^{3} J_{\mathrm{Ha}, \mathrm{H}}=7.2 \mathrm{~Hz},{ }^{3} J_{\mathrm{Hb}, \mathrm{H}}=7.6 \mathrm{~Hz}$, $\left.2 \mathrm{H}, \mathrm{CH}_{2} \mathrm{CO}\right), 3.14-3.20\left(\mathrm{~m}, 4 \mathrm{H}, \mathrm{CH}_{2}-\mathrm{NH}\right), 4.91-5.02\left(\mathrm{~m}, 12 \mathrm{H},-\mathrm{CHOCO}+\mathrm{CH}_{2}=\mathrm{CH}-+\mathrm{N} \underline{\mathrm{H}}\right)$, 5.74-5.88 (dxdxt, $\left.{ }^{3} J_{\mathrm{H}, \mathrm{H}}=6.6 \mathrm{~Hz},{ }^{3} J_{\mathrm{H}, \mathrm{H} \text { cis }}=10.4 \mathrm{~Hz},{ }^{3} J_{\mathrm{H}, \mathrm{H} \text { trans }}=17.1 \mathrm{~Hz}, 4 \mathrm{H}, \mathrm{CH}_{2}=\mathrm{CH}-\right) .{ }^{13} \mathrm{C} \mathrm{NMR}$ (75.6 MHz, $\left.\mathrm{CDCl}_{3}\right): \delta_{\mathrm{C}} 23.2,25.3,26.3,29.0,29.2,29.3,29.4,30.8\left(\underline{\mathrm{CH}}_{2}\right), 33.9\left(\mathrm{CH}_{2}=\mathrm{CH}-\underline{\mathrm{CH}}_{2}\right)$, $38.7\left(\underline{\mathrm{CH}}_{2}-\mathrm{CO}\right), 41.0\left(\underline{\mathrm{CH}}_{2}-\mathrm{NH}\right), 78.6(\underline{\mathrm{CHOCO}}), 114.2\left(\underline{\mathrm{CH}}_{2}=\mathrm{CH}\right), 139.3\left(\mathrm{CH}_{2}=\underline{\mathrm{CH}}\right), 156.0$ (OCCONHR), 209.0 (드). IR (ATR, v, $\mathrm{cm}^{-1}$ ): 3325 (sharp, m, NH), 3077 (w, $\left.\mathrm{CH}_{2}=\mathrm{CH}\right), 2923$ (vmax, alkyl), 2851 (s, alkyl), 1716 (w, CO), 1694 (s, CO), 1642 (w, $\mathrm{CH}_{2}=\mathrm{CH}$ ). MS (+ESI, 4000 $\mathrm{V}): m / z(\%)=505(\mathrm{M}+1-1 \mathrm{x}$ acyloin side chain $(336), 100), 841(\mathrm{M}+1,62)$. HRMS (+ESI) calcd for $\mathrm{C}_{52} \mathrm{H}_{93} \mathrm{~N}_{2} \mathrm{O}_{6}\left(\mathrm{M}^{+}+1\right)$ : 841.70282; found: 841.70176 .

General procedure for the reaction of acyloin 1 with bifunctional 2,4-toluene diisocyanate To a flame dried $50 \mathrm{~mL}$ flask a solution of acyloin $(1.35 \mathrm{~g}, 4.0 \mathrm{mmol})$ in extra dry 1,2dichloroethane (dried with molecular sieves, $15 \mathrm{~mL}$ ) was added and placed under nitrogen gas. Then 2,4-toluene diisocyanate $(0.35 \mathrm{~g}, 2 \mathrm{mmol}, 0.50 \mathrm{eq})$ was added by means of a syringe through a septum. The reaction mixture was refluxed for $3.5 \mathrm{~h}$. In ${ }^{1} \mathrm{H}$ NMR a conversion of more than $95 \%$ was observed. After evaporation of the solvent, product 4 was purified by column chromatography on silica with 10:1 petroleum ether: ethyl acetate to 3:1 petroleum ether: ethyl acetate.

Bis(12-oxodocosa-1,21-dien-11-yl) 2,4-toluene dicarbamate (4). Pale yellow viscous oil, yield $22 \%, 0.38$ g. ${ }^{1} \mathrm{H} \mathrm{NMR}\left(300 \mathrm{MHz}, \mathrm{CDCl}_{3}\right): \delta_{\mathrm{H}} 1.28-1.79\left(\mathrm{~m}, 52 \mathrm{H}, \mathrm{CH}_{2}\right), 2.00-2.06(\mathrm{~m}, 8 \mathrm{H}$, $\left.\mathrm{CH}=\mathrm{CH}_{2}-\underline{\mathrm{C}}_{2}\right), 2.23\left(\mathrm{~s}, 3 \mathrm{H}, \mathrm{CH}_{3} \mathrm{Ar}\right), 2.34-2.55\left(\mathrm{~m}, 4 \mathrm{H}, \mathrm{C}_{2} \mathrm{CO}\right), 4.90-5.10(\mathrm{~m}, 10 \mathrm{H},-\mathrm{CHOCO}$ $\left.+\mathrm{CH}_{2}=\mathrm{CH}-\right), 5.74-5.87\left(\mathrm{dxdxt},{ }^{3} J_{\mathrm{H}, \mathrm{H}}=6.6 \mathrm{~Hz},{ }^{3} J_{\mathrm{H}, \mathrm{H}}\right.$ cis $=10.2 \mathrm{~Hz},{ }^{3} J_{\mathrm{H}, \mathrm{H}}$ trans $=17.1 \mathrm{~Hz}, 4 \mathrm{H}$, $\left.\mathrm{CH}_{2}=\mathrm{CH}-\right), 6.61(\mathrm{~s}, 1 \mathrm{H}, \mathrm{NH}), 6.83(\mathrm{~s}, 1 \mathrm{H}, \mathrm{NH}), 7.07-7.10$ (m, $\left.\underline{\mathrm{C}}_{\text {arom }}\right), 7.13-7.23$ (m, $\left.\underline{\mathrm{C}}_{\text {arom }}\right)$, 7.75-7.77 (m, $\left.\underline{\mathrm{C}}_{\text {arom}}\right) .{ }^{13} \mathrm{C} \mathrm{NMR}\left(75.6 \mathrm{MHz}, \mathrm{CDCl}_{3}\right): \delta_{\mathrm{C}} 17.1\left(\mathrm{CH}_{3} \mathrm{Ar}\right), 23.16,23.20,25.3,29.0$, 29.17, 29.25, 29.38, 29.42, 29.45, 30.7, $30.8\left(\underline{\mathrm{CH}}_{2}\right), 33.9\left(\mathrm{CH}_{2}=\mathrm{CH}-\underline{\mathrm{CH}}_{2}\right), 38.7,38.8\left(\underline{\mathrm{CH}}_{2}-\mathrm{CO}\right)$, 79.0, 79.1 (태OCONHAr), $114.3\left(\underline{\mathrm{CH}}_{2}=\mathrm{CH}\right), 130.9$ ( $\left.\underline{\mathrm{CH}}_{\text {arom. }}\right), 135.9,136.4$ ( $\left.\underline{\mathrm{C}}_{\text {arom.quat. }}\right), 139.3$ $\left(\mathrm{CH}_{2}=\underline{\mathrm{C}} \mathrm{H}\right.$ ), 152.9, 153.1 (OCOONHAr), 208.3, 208.6 (으). IR (ATR, v, $\mathrm{cm}^{-1}$ ): 3320 (broad, w, $\mathrm{NH}), 3075$ (w, $\mathrm{CH}_{2}=\mathrm{CH}$ ), 2924 (v $\max$, alkyl), 2853 (s, alkyl), 1718 (s, CO), 1640 (w, $\mathrm{CH}_{2}=\mathrm{CH}$ ), $1600\left(\mathrm{w}, \mathrm{C}_{6} \mathrm{H}_{5}\right)$. MS (-ESI, $\left.4000 \mathrm{~V}\right): \mathrm{m} / \mathrm{z}(\%)=509$ (M-1- 1x acyloin side chain (336), 100) ,845 $(\mathrm{M}-1,14), 881\left(\mathrm{M}-1+2 \mathrm{xNH}_{4}^{+}(36), 10\right)$. HRMS (+ESI) calcd for $\mathrm{C}_{53} \mathrm{H}_{90} \mathrm{~N}_{3} \mathrm{O}_{6}\left(\mathrm{M}^{+}+\mathrm{NH}_{4}^{+}\right)$: 864.682; found: 864.683 .

Mono-(2-Oxazolidinone) derived from (4). Yield 61\%, $1.04 \mathrm{~g} .{ }^{1} \mathrm{H} \mathrm{NMR}\left(300 \mathrm{MHz}, \mathrm{CDCl}_{3}\right): \delta_{\mathrm{H}}$ $1.0-1.9\left(\mathrm{~m}, 54 \mathrm{H}, \underline{\mathrm{C}}_{2}\right), 1.9-2.1\left(\mathrm{~m}, 8 \mathrm{H}, \mathrm{CH}=\mathrm{CH}_{2}-\mathrm{C}_{2}\right), 2.1-2.3\left(\mathrm{~m}, 3 \mathrm{H}, \mathrm{Ar}-\underline{\mathrm{H}}_{3}\right), 2.4-2.6$ (m, $2 \mathrm{H}, \underline{\mathrm{C}}_{2} \mathrm{CO}$ ), 4.2-4.4 (m, 1H, $\left.\underline{\mathrm{C}}_{\text {oxazolidinone }}\right), 4.9-5.1$ (m, 9H, $\underline{\mathrm{H}}_{2}=\mathrm{CH}-+\underline{\mathrm{CHOCONHAr}}$ ), 5.7-5.9 (m, 4H, $\left.\mathrm{CH}_{2}=\mathrm{C} \underline{\mathrm{H}}-\right)$, 7.0-7.8 (m, 3H, $\left.\underline{\mathrm{C}}_{\text {arom }}\right)$. 


\section{References}

1. www.biohub.fr

2. Van Haveren, J.; Oostveen, E. A.; Miccichè F.; Weijnen J. G. J. 227th National Meeting of the American Chemical Society, Anaheim, CA, March 28-April 01, 2004. (also published as: Van Haveren, J.; Oostveen, E. A.; Miccichè F.; Weijnen J. G. J. In Feedstocks for the future: Renewables for the production of chemicals and materials; Bozell J.J.; Patel M.K. Eds.; American Chemical Society: Washington, 2006; Vol 921, p 99.)

3. Feng, X. H.; Jaffe, M.; Hammond, W. B.; East, A. J. 35th Annual Northeast Bioengineering Conference, Cambridge, MA, April 3-5, 2009, 175-176.

4. Van der Steen, M.; Stevens, C. V. ChemSusChem 2009, 2, 692.

5. Van der Steen, M.; Stevens, C. V.; Eeckhout, Y.; De Buyck, L.; Ghelfi, F.; Roncaglia, F. Eur. J. Lipid Sci. Technol. 2008, 110, 846.

6. Nguyen, My T.; Doan, V.; Kwo, K.; Bui, L. V. U. S. Patent 6,395,811, 2002; Chem. Abstr. 2002, 136, 402556.

7. Stephens, W. D.; Rodman, B. K. U. S. Patent 4,482,408, 1985; Chem. Abstr. 1985, 102, 187496.

8. Griffiths, M. J.; Hall, S. A., Ivory, N. E. PCT Int. Appl. 94/14902, 1995; Chem. Abstr. 1995, 123, 12046.

9. Langley, R.; Robertson, G. H.; Bridge, C. J. Ger. Offen. 2350454, 1972; Chem. Abstr. 1974, $81,137674$.

10. (a) Iyengar, S. S. R.; Ganpat, R. K.; Kumar, S. P.; Swaminathan, S. U.S. Patent 5,952,411, 1999; Chem. Abstr. 1999, 131, 200832. (b) Ramaswamy, S. S.; Ganpat, R. K.; Kumar, S. P.; Swaminathan, S. IN188825, 2002; Chem. Abstr. 2006, 145, 455632.

11. Satgé, C.; Granet, R.; Verneuil, B.; Champavier, Y.; Krausz, P. Carbohydr. Res. 2004, 339, 1243.

12. Kušan, J.; Keul, H.; Höcker, H. Macromol. Symp. 2001, 165, 63.

13. Martínez, R.; Jiménez-Vázquez, H. A.; Tamariz, J. Tetrahedron 2000, 56, 3857.

14. 14.Chernysheva, N. B.; Bogolyubov, A. A.; Semenov, V. V. Chem. Heterocycl. Compd. 1999, 35, 216.

15. Gompper, R. Chem. Ber. 1956, 89, 1748.

16. Easton, N. R.; Cassady, D. R.; Dillard, R. D. J. Org. Chem. 1962, 27, 2927.

17. Francis, T.; Thorne, M. P. Can. J. Chem. 1976, 54, 24.

18. Santoyo, B. M.; González-Romero, C.; Merino, O.; Martínez-Palou, R.; Fuentes-Benites, A.; Jiménez-Vázquez, H. A.; Delgado, F.; Tamaríz, J. Eur. J. Org. Chem. 2009, 2505. 